FORMATION Formation emploi

Revue française de sciences sociales

124 | Octobre-Décembre 2013

Pêle-mêle

\title{
Les cheminements des étudiants vers l'insertion professionnelle : entre se « placer » et se «trouver »
}

Les cheminements des étudiants vers l'insertion professionnelle : entre se

"placer » et se " trouver»

Pathways towards job placement. The students between two logics:

"integration" and "self accomplishment"

Wege von Studierenden zur beruflichen Eingliederung: zwischen „Platz finden“

und "Sich finden"

Los recorridos de los estudiantes hacia la inserción profesional : entre

"colocarse" $y$ "encontrarse"

\section{Emmanuelle Maunaye}

\section{(2) OpenEdition}

Journals

Édition électronique

URL : http://journals.openedition.org/formationemploi/4072

DOI : $10.4000 /$ formationemploi.4072

ISSN : 2107-0946

Éditeur

La Documentation française

Édition imprimée

Date de publication : 31 décembre 2013

Pagination : 7-22

ISSN : 0759-6340

Référence électronique

Emmanuelle Maunaye, «Les cheminements des étudiants vers l'insertion professionnelle : entre se " placer » et se « trouver » », Formation emploi [En ligne], 124 | Octobre-Décembre 2013, mis en ligne le 08 janvier 2015, consulté le 30 octobre 2020. URL : http://journals.openedition.org/formationemploi/ 4072 ; DOI : https://doi.org/10.4000/formationemploi.4072 


\section{Les cheminements des étudiants vers l'insertion professionnelle: entre se " placer » et se « trouver »}

Emmanuelle Maunaye Maître de conférences en sociologie à l'université de Rennes 1, Département des Carrières Sociales (IUT - Institut universitaire de technologie). Chercheure au CRAPE (Centre de Recherches sur l'Action Politique en Europe). Thèmes de recherche: la jeunesse étudiante; la mobilité, le logement, les relations à la famille, le parcours d'études et l'insertion professionnelle

Résumé

Les cheminements des étudiants vers l'insertion professionnelle : entre se « placer » et se « trouver»

Le sens et les représentations que les étudiants se forgent de leurs études et de leur projet d'orientation et d'insertion professionnelle sont au cœur de cet article. Les étudiants se différencient selon leur vision de l'avenir, qu'ils aient ou non déterminé un projet futur. En nous centrant sur les étudiants "indéterminés ", nous chercherons à montrer que cette indétermination peut être analysée comme la marque d'une tension entre la logique du placement et la logique de la réalisation de soi ; cette tension ayant également des effets sur les étudiants " déterminés ».

Mots clés : cheminement universitaire, insertion professionnelle, projet, orientation scolaire-professionnelle

Abstract

Pathways towards job placement. The students beetween two logics:

« integration » and « self accomplishment »

This article deals with the value and the representation that the students have of their studies, their career plan and their integration into the world of work. The students differentiate themselves from each other according to their vision of the future, depending on their future prospects. Through the analysis of the "undecided" students, we will try to point out to what extent this indecisiveness may reveal the signs of a tension between a logic of job integration and that of self-accomplishment. This tension also influences the "determined" students.

Keywords: undergraduate paths, transition from school to work, project, school guidance $\&$ vocational guidance

Journal of Economic Literature: I 29, J 24

Traduction : Auteurs 
Aujourd'hui, en Europe en général et en France en particulier, les jeunes sont confrontés à une précarisation grandissante de leurs parcours d'insertion professionnelle en raison de situations socio-économiques de plus en plus dégradées, y compris chez les jeunes diplômés de l'enseignement supérieur.

Les enquêtes "Génération » du Céreq observent que les parcours d'entrée dans la vie professionnelle des jeunes diplômés se complexifient : périodes d'attente, de chômage, d'emplois précaires se superposent avant une stabilisation professionnelle retardée. Ces jeunes diplômés sont également plus souvent qu’auparavant touchés par des phénomènes de déclassement, si l'on considère leurs difficultés croissantes à obtenir des emplois en rapport avec leur niveau de qualification (Giret, 2009). Néanmoins, toutes les enquêtes sur l'insertion des jeunes montrent "qu'un diplôme de l'enseignement supérieur est un atout sur le marché du travail alors que l'absence de diplôme semble être un handicap de plus en plus lourd" (Giret, 2009, p. 331). Néanmoins, tous les diplômes de l'enseignement supérieur ne "protègent " pas de la même manière des risques de précarité et de déclassement. "À niveau de diplômes équivalents, ceux issus de filières professionnelles entrent dans la vie active dans de meilleures conditions que les diplômés des filières générales" (Erlich et Verley, 2010).

Dans ce contexte, la lutte contre les sorties précoces et sans diplôme de l'université est devenue un axe majeur des politiques des établissements de l'enseignement supérieur. Ces politiques universitaires visent à assurer un meilleur accompagnement des étudiants dans leur formation et la construction de leur projet d'orientation et d'insertion professionnelle. Le plan « réussite en licence ", initié par Valérie Pécresse en 2008, a été conçu en ce sens.

Face à ces circonstances considérées comme difficiles pour bon nombre de jeunes, quel sens et quelles représentations les étudiants forgent-ils de leurs études et de leur future insertion professionnelle ? Cette question sera au cœur de cet article. En la replaçant dans le champ des analyses sur la jeunesse et le passage à l'âge adulte, période de la vie définie comme une phase de construction de soi, d'expérimentations, on prêtera attention à la manière dont ces jeunes construisent et anticipent leur parcours non seulement professionnel mais aussi social.

L'insertion professionnelle est ici envisagée comme un processus. Nous cherchons à comprendre les cheminements suivis par les étudiants, cheminements traversés d'incertitudes, de difficultés, de doutes. Précisément, nous préférons parler de cheminements plutôt que de trajectoires, et nous rejoignons en cela les réserves qu'émet Claire Bidart (2006) à l'égard de cette notion qui induit souvent l'idée d'un itinéraire "balisé, ordonné et prévisible» (p. 30). C'est bien souvent la mise en récit, par l'acteur social lui-même, de ses expériences passées, qui élabore le sens de la trajectoire et des liens entre les étapes ou les événements qui y sont inclus. Notre approche est ici différente. 
Notre objectif est d'interroger les étudiants sur la manière dont ils " construisent » leur chemin, en leur demandant de se tourner non plus vers le passé mais vers l'avenir.

L'examen des trajectoires d'insertion scolaire et professionnelle des jeunes en Europe montre clairement des disparités nationales. La France fonctionne sur un modèle particulier où l'intégration sociale est dominée par l'enjeu des études et du premier emploi (Van de Velde, 2008). L'identité socioprofessionnelle est ainsi principalement déterminée par le niveau d'études et le diplôme initial, qui dépendent eux-mêmes de l'origine sociale notamment.

La jeunesse est vécue alors comme la période du "placement » au sein d'une hiérarchie prédéfinie, durant laquelle les choix opérés (orientation, temps des études, ...) ont un retentissement considéré comme quasi définitif sur la trajectoire à venir. Le choix des études devient ainsi un enjeu majeur : on considère toujours que la formation initiale et le diplôme obtenu qui y correspond orientent de manière fondamentale et irréversible la position socioprofessionnelle future de l'individu. Dans ce contexte, les parcours d'études et d'insertion socioprofessionnelle, linéaires et déterminés précocement, sont valorisés.

Dès lors, comment les étudiants construisent-ils leurs projets futurs ?

Dans un premier temps, nous envisagerons la manière dont les étudiants se représentent leur future insertion professionnelle, en insistant sur la dimension temporelle de la question. Ces derniers envisagent leur sortie des études et leur entrée sur le marché du travail de façon lointaine. Dans un deuxième temps, nous montrerons que les étudiants se différencient selon leur vision de l'avenir, qu'il aient ou non déterminé un projet futur. En nous arrêtant, dans un troisième temps, sur les étudiants indéterminés, nous chercherons à montrer que cette indétermination peut être analysée comme la marque d'une tension entre la logique du placement et la logique de la réalisation de soi ; tension qui a également des effets sur les étudiants déterminés. 


\section{Encadré 1}

\section{L'enquête}

Les analyses réalisées sont issues d'une recherche menée à l'université de Tours entre 2009 et 2010. Initiée par la présidence de l'établissement, préoccupée par l'enjeu de l'insertion professionnelle de ses diplômés et la réalité de la prise en compte de ces questions sur le terrain, elle visait l'identification des intérêts et des attentes des étudiants ainsi que des enseignants-chercheurs $\left(^{*}\right)$ en la matière.

Méthodologiquement, cette recherche d'approche compréhensive a utilisé la technique de l'entretien semi-directif pour le recueil des données. L'objectif était donc d'approcher les représentations et les stratégies des différents acteurs et de comprendre la manière dont elles étaient produites. Le travail présenté ici se centre exclusivement sur les données recueillies auprès des étudiants.

Nous avons choisi de rencontrer les étudiants à un moment précis de leur parcours universitaire. Tous étaient inscrits à un niveau de troisième année d'études supérieures. Celle-ci a été choisie comme une année charnière où les questions de choix de poursuite d'étude et d'insertion professionnelle sont supposées se poser de façon saillante. Des étudiants inscrits dans des filières longues constituent notre population. Nous leur avons demandé de revisiter leur trajectoire jusqu'à leur entrée dans l'enseignement supérieur et de révéler leurs projets d'orientation et d'insertion futurs. Les étudiants inscrits dans des filières courtes et professionnalisantes ne constituaient pas notre population cible. En effet, ces formations sont construites en référence à un (ou des) métier(s) précis et recrutent leurs étudiants en fonction de leurs motivations à exercer ce métier. Dans notre enquête, nous souhaitions rencontrer des étudiants inscrits dans des filières générales, aux débouchés plus larges ou souvent moins bien identifiés.

Chaque entretien a duré environ une heure et a été retranscrit intégralement. Les discours recueillis ont été analysés dans une perspective comparative, en référence à plusieurs thèmes, objets de notre questionnement : le parcours suivi par l'étudiant depuis son entrée à l'université, ses objectifs et ses motivations lors de l'entrée ; ses objectifs et ses motivations aujourd'hui ; les réajustements et réorientations dans le parcours ; la connaissance et l'usage des ressources à l'intérieur comme à l'extérieur de l'université ; le métier et l'insertion professionnelle envisagés : connaissance du secteur, du métier visé, adéquation entre le projet et sa mise en œuvre par les études, représentations du milieu professionnel, du monde du travail ; les ressentis face à la future insertion professionnelle; la place de l'université et de ses services dans l'insertion professionnelle.

Au final, notre corpus est constitué de 43 entretiens d'étudiants - hommes et femmes dans une proportion quasi égale. Nous avons cherché à privilégier la diversité des filières : des étudiants inscrits dans les filières des différentes composantes de l'université François Rabelais de Tours ont été rencontrés : Arts et Sciences Humaines (ASH), Lettres et Langues, Droit, Sciences, la faculté de médecine et l'école Polytechnique de l'université. 


\section{Suite encadré 1}

$\left.{ }^{*}\right)$ : En contrepoint des discours étudiants, il était intéressant d'approcher les représentations enseignantes concernant ces enjeux de l'insertion professionnelle, précisément en ces temps de mutations de l'enseignement supérieur où la question du devenir des diplômés devient un enjeu majeur. Des entretiens auprès de 12 enseignants-chercheurs, responsables de licence (UFR - Unité de formation par la recherche - Lettres et Langues ; Arts et Sciences Humaines ; Droit, Economie et Sciences Sociales ; Sciences et Techniques) ont été réalisés entre mars et juin 2008. L'objectif de ces rencontres était d'interroger ces enseignants sur les manières dont euxmêmes - mais aussi les équipes pédagogiques dont ils font partie - concevaient les enjeux de I'insertion professionnelle des étudiants inscrits dans leur filière.

\section{L'insertion professionnelle : un horizon encore lointain}

Interrogés au cours de la troisième année de leurs études supérieures, les étudiants rencontrés donnent l'image d'être " au cœur " de leurs études. Quelle que soit la filière suivie, aucun d'entre eux n'envisage de mettre un terme à sa scolarité à la fin de l'année en cours. Pour les étudiants inscrits en faculté de médecine ou à l'école d'ingénieurs Polytechnique de l'université, cela n'aurait pas de sens. Pour ces deux types de filière, la troisième année d'études ne délivre pas de diplôme précis, valorisable sur le marché professionnel'

Pour les étudiants des filières générales, ces licences ne sont pas présentées comme préparant à une professionnalisation dans l'optique d'une insertion immédiate mais comme s'inscrivant dans une poursuite d'études en master. Sur ce point, les étudiants l'ont bien compris et leurs comportements sont conformes à ce qui est préconisé.

La fin du cursus est donc un horizon certes envisagé mais lointain, tout comme l'insertion professionnelle qui en découle. À ce titre, les étudiants avouent des difficultés à s'imager les conditions de leur insertion professionnelle : "Vu que c'est dans super longtemps quion va vraiment travailler, quion va vraiment être livré à nous-mêmes. Je ne sais pas si je peux bien répondre maintenant » explique Sandra (22 ans, DCEM, Deuxième Cycle d'Etudes Médicales 1). Concrètement, il est certes très difficile pour ces jeunes de concevoir leur avenir étant donné le contexte socio-économique actuel du pays, la situation difficile de leur génération face à l'emploi, les perspectives incertaines.

1. Les études médicales, d'une durée minimale de neuf années, sont réparties en trois cycles (qui ne sont pas adossés au système LMD - licence, master, doctorat - comme dans le reste de l'université). La troisième année d'études de médecine correspond à la première année du deuxième cycle. De la même manière, les étudiants inscrits à l'école Polytechnique viennent seulement d'entamer leur formation d'ingénieur dont la sortie est située à bac +5 . 
Plus fondamentalement, cette attitude traduit aussi une représentation des parcours d'insertion socioprofessionnelle typique du modèle français. L'intégration sociale est dominée par l'enjeu des études et du premier emploi, ce qu’on a appelé, précédemment, "la logique du placement " (Van de Velde, 2008). Ce modèle contribue à envisager une "conception ternaire des trajectoires : études non-salariées, période d'insertion, puis accès à l'emploi " où les périodes se succèdent plus qu'elles ne se mêlent. Cette période d'insertion est d'autant moins envisagée qu'elle est séparée de celle des études par "une frontière ", "un fossé ». Ces représentations selon lesquelles il y aurait une séparation nette entre deux périodes du cycle de vie soutiennent l'idée selon laquelle ces jeunes pensent le cours de l'existence comme une succession d'étapes à franchir, l'intégration sociale et professionnelle faisant partie de celles-là : "Ça va être une nouvelle étape dans ma vie. " (Lucie, 23 ans, L3 Droit-Langues)

"Temps des études et temps de l'emploi sont ainsi vécus séparément" (Van de Velde, 2008, p. 145) rendant prématurée toute projection des conditions de l'avenir. "Pour le moment, je suis dans les études " explique Simon (20 ans, L3 de droit). "Pour l'instant, je n'ai pas d'idée, je suis encore dans mes études, donc je travaille à fond là-dedans " renchérit Estelle (21 ans, L3 de droit). "Pour moi, c'est l'inconnu en fait. Je ne vois pas du tout, je n'arrive pas à me projeter à ce moment-là » (Alexandre, 24 ans, L3 biologie).

\section{Deux visions de l'avenir : avoir ou non un projet déterminé}

Les étudiants se rejoignent sur cette volonté de poursuivre leurs études et la difficulté à dessiner des représentations claires de leurs conditions d'insertion professionnelle ; en revanche, ils divergent sur les raisons pour lesquelles ils ont entamé leurs études actuelles et sur la présence ou non d'un projet professionnel connecté. Deux grands profils d'étudiants se dessinent alors : les étudiants ayant déterminé un projet d'insertion et les étudiants indéterminés, n’ayant pas encore élaboré de projet.

Les premiers, les étudiants au projet déterminé sont capables de citer un métier ou un champ d'activités pressenti : le choix de la filière d'études dans laquelle ils sont engagés étant directement relié à cet objectif. À l'inverse, les seconds, les étudiants sans projet déterminé n'identifient pas de métier ou de champs d'activités précis. Le choix des études n'est pas en relation avec un projet professionnel particulier. Si certains d'entre eux peuvent néanmoins citer quelques dimensions professionnelles, elles n’ont pas de liens entre elles et dénotent un manque de perspectives affirmées.

\subsection{Les étudiants au projet déterminé}

Ce groupe d'étudiants est constitué de jeunes qui se sont déterminés un projet professionnel et qui ont identifié le plus souvent un métier. Dans notre corpus, il s'agit des étudiants inscrits en faculté de médecine et à l'école Polytechnique de l'université mais aussi 
de certains étudiants inscrits dans des filières générales : le droit, les langues, les lettres, les sciences humaines et sociales.

Pour les étudiants inscrits en médecine et à l'école Polytechnique, l'attitude n'est pas étonnante si l'on considère que l'entrée dans ces formations s'effectue sur concours ; des concours très sélectifs qui exigent une motivation importante quelquefois ancienne : « $\grave{A}$ partir du moment où j'ai su parler, j’ai toujours dit que je voulais être médecin " (Noémie, 21 ans, DCEM1). Alice souhaite devenir ingénieure en infographie : "J’y pense depuis une dizaine d'années. Cette idée m'est venue par gồt pour l'informatique, pour mettre à profit mon esprit créatif dans un domaine qui m’attire particulièrement : le cinéma. " (20 ans, école polytechnique)

Pour les étudiants inscrits dans un cursus général, le choix du métier a présidé au choix de la filière. Olivier (22 ans) voudrait embrasser la carrière de journaliste et les études de sociologie lui semblent être les plus adaptées pour préparer les concours aux écoles. Pigiste depuis l'âge de 16 ans, accumulant les expériences, il se perçoit déjà comme un professionnel dont la reconnaissance institutionnelle ne peut être acquise que par le diplôme : "C'est un peu un frein parce qu'on est dans une société en France où l'on base tout sur le diplôme. " Le choix du métier peut être également plus récent, postérieur à l'entrée dans l'enseignement supérieur : la formation elle-même, des rencontres avec des professionnels, des conférences métiers ayant contribué à définir le projet.

Pour ces jeunes, l'avenir et l'insertion professionnels sont envisagées avec confiance. La bonne réputation des études poursuivies, en partie due à l'excellente employabilité des étudiants sortis de ces formations, suffit pour rester serein. Les études de médecine « assurent » un débouché. L'école Polytechnique développe une politique très volontariste en matière de placement de ses étudiants : "Même un peu trop, je trouve. En fait, à polytech', ils ont tellement d'accord avec le privé quil y a un moment où tu te demandes si tu travailles encore pour toi et pour ton savoir parce quils ont au moins quarante accords avec des entreprises dans tous les domaines. " (Loïc, 23 ans, école Polytechnique)

Dans les filières générales où les débouchés sont souvent considérés comme inexistants, où les filières ne sont pas valorisées, le fait même d'avoir su déterminer un projet et de s'y tenir permet d'éclaircir l'avenir. Ils sont ainsi conformes à une norme valorisée et attendue : la détermination précoce des choix d'orientation et d'insertion professionnelle (Van de Velde, 2008).

\subsection{Les étudiants sans projet : ceux qui ne savent pas... ou qui ne savent plus}

Dans notre corpus, ce groupe d'étudiants est constitué de jeunes exclusivement inscrits dans les filières générales de l'université : sciences, sciences humaines et sociales, lettres, langues. Le processus d'entrée à l'université et la dynamique du choix de la filière expliquent en partie cette situation. Ceux qui ont choisi par défaut ne sont pas rares. Ils ont été refusés 
dans des filières sélectives dés l'entrée ou en cours de cursus : l'inscription en filière générale a constitué ainsi une porte de sortie. Clément a entamé des études d'infirmier dés la sortie du lycée. Ses résultats ne lui ont pas permis de poursuivre sa formation à l'issue de la première année. Le verdict étant intervenu tardivement, il a eu peu de temps pour choisir une orientation : "Il me restait à peu près que quatre ou cinq jours pour pouvoir minscrire à la fac. Donc là, ça a été très vite. Je me suis dit 'Bon, qu'est-ce qui me plaît? en quoi jétais à peu près bon au lycée?'. Et c'est l'anglais qui est sorti du lot. Donc au final, j'ai dit 'Pourquoi pas? On va tenter la fac d'anglais et puis on va voir si ça marche, comment je peux gérer ça et ce vers quoi ça va m’amener." " (21 ans, L3 anglais) Marie, quant à elle, affirme avoir "choisi la socio par hasard ». En terminale, son projet était pourtant affirmé : après un bac $S$, elle était acceptée dans une école de chiropracteur mais, étonnamment, elle refuse : "C'est marrant : quand je rêvais d'y aller, je pensais que je n'y arriverais jamais et quand j'ai enfin pu y accéder, là c'est moi qui ne voulais pas. C'est marrant la vie. "(20 ans, L3 sociologie)

Pour certains, l'entrée dans les études supérieures a pu aussi mettre en doute l'orientation choisie initialement. Par exemple, cet étudiant inscrit en L3 d'anglais hésite tour à tour entre se diriger dans la voie du professorat, ou bien dans celle de l'aéronautique comme steward dans une compagnie aérienne ou bien encore, dans la voie médicale : ce dernier choix lui demanderait de reprendre ses études depuis le début. À bien des égards, cet étudiant « ne sait plus »: un choix professionnel se dessinait lors de son entrée à l'université (le professorat). Il lui a permis de choisir sa filière d'études, mais depuis tout s'est emmêlé. Son projet est dans l'impasse. Cette attitude a été observée chez d'autres étudiants de notre corpus. Elle constitue une variante dans le groupe des étudiants indéterminés.

Qu'ils soient dans l'urgence ou non, que l'entrée à l'université en filière générale soit un premier choix ou non, les étudiants "qui ne savent pas " ont en commun d'avoir choisi une orientation non pas en vue d'un métier précis mais en fonction de l'attrait qu'ils avaient pour la matière, en référence à leurs expériences du lycée. Grégoire s'est inscrit en Histoire "par goût, même si jétais conscient que les débouchés étaient minces" (21 ans). La passion pour la discipline, l'envie d'enrichir ses connaissances et de s'enrichir personnellement ont guidé son choix. Il est typiquement dans les études, l'insertion professionnelle n'étant pas à l'ordre du jour : "Je n'ai pas encore l'optique d'être dans la vie professionnelle. J'aime bien les études, ça me plaît d'apprendre des choses, mais je ne vois pas beaucoup plus loin. " Guidée par l'attrait de la matière et le goût pour les études, l'expérience universitaire en dehors d'un projet professionnel construit n'est donc pas mal vécue.

Pour ces étudiants sans projet, les échéances, le temps du choix et de la détermination sont repoussés à plus tard. Cette indétermination dans la construction de son parcours se comprend comme une position d'attente, destinée à laisser le champ des opportunités le plus ouvert possible. Sous-ensemble de ces étudiants " qui ne savent pas ", l'attitude des étudiants « qui ne savent plus » est, à cet égard, éclairant. L'épreuve des études a modifié leur projection, a suscité des intérêts nouveaux qu'ils ne parviennent pas à départager. Antony (20 ans), qui achève sa L3 d'anglais, s'interroge beaucoup : "Là je suis censé partir un an 
à l'étranger et à mon retour, je me demande si je ne vais pas tout recommencer à zéro et partir sur des études de médecine. "Tout son entretien est marqué par l'examen des différentes possibilités qui pourraient s'offrir à lui : "J'aime bien ce que je fais, il n'y a pas de problème mais dans l'idéal, jaime bien toucher à tout. Si je pouvais faire cinq métiers dans ma vie, je le ferai (...). Oui, si j'avais pu être juriste, je l'aurais fait. Si j'avais pu être prof, enfin c'est ce que je compte faire... Si javavis pu être médecin... ". En fin d'entretien, il évoque aussi la tentation "d'intégrer une boîte de personnels navigants commerciaux pour devenir steward".

La fin du premier cycle n'est pas encore propice au questionnement d'ordre professionnel. Ces décisions sont reportées à un an (M1) voire deux ans (M2); sans pour autant que l'étudiant explique quelles " stratégies " il pense mettre en œuvre pour se déterminer. Lattente et l'indécision, contraires aux injonctions normatives appelant la détermination précoce du projet, sont converties en accumulation de diplômes, fondée sur la justification que les diplômes protègent du chômage, comme l'exprime Charlotte (21 ans, L3 de psychologie) : "Pour moi, les études, ça correspond à une sûreté. Avoir un diplôme donc avoir des débouchés."

Elles traduisent néanmoins des sentiments très ambivalents à l'égard de la sortie de l'université et de l'insertion dans le monde du travail. "Linquiétude», "lincertitude», "le pessimisme ", "l'angoisse", "le doute, la peur et le stress " s'entendent dans les discours recueillis. Ces sentiments de malaise sont nourris par la peur de la précarité, qu’elle soit financière ou professionnelle, ainsi que par une représentation négative du monde du travail, jugé comme menaçant. Pour Emilie (21 ans, L3 de sociologie), "Le monde du travail est un monde difficile. Il faut trouver sa place, faire ses preuves. On est jugé en permanence. C'est du stress, on joue sa vie quand même". "J'imagine le monde du travail comme un monde qui ne fait pas de cadeau: marche ou crève. Il y a beaucoup de concurrence, beaucoup de gens qui ont les dents longues" (Anaïs, 20ans, L3 de sociologie). "C'est la jungle, dans les petits boulots d'été, j'ai pu voir que c'était quand même un peu du chacun pour soi " (Coralie, 20 ans, L3 de psychologie).

L'enquête récente "Les jeunesses face à leur avenir » (Stellinger, 2008), qui compare les jeunesses de huit pays, souligne clairement que les jeunes français font partie de ceux qui sont les plus pessimistes au sujet de leur intégration sociale et professionnelle (avec le Japon, l'Espagne et l'Allemagne). Ils se disent peu certains d'avoir un "bon travail" et un "avenir prometteur" (de Singly, 2008). "Notre génération, de toute façon, va être plus que précaire. On va travailler toute notre vie et on va en chier à l'infini. Alors si tu commences à te projeter là-dedans, tu te prends la tête à l'infini. Toutes ces questions, je me les pose tous les soirs en m'endormant. Je suis limite insomniaque parce que je me demande "Qu'est-ce que tu vas faire de ta vie ?" et je n'ai pas de réponse " (Alexandre, 25 ans, L3 biologie).

En même temps qu'ils énoncent les sources de leurs angoisses, les étudiants peuvent aussi se dire confiants sur leur propre avenir lié eu égard à leur capacité de débrouillar- 
dise et à la réalisation de leur indépendance personnelle. Pour Olivier Galland (2009), il s'agit avant tout "d'une crise de confiance collective " avant une crise de confiance individuelle. Jean-Claude Richez, pour sa part, évoque l'existence d'un « véritable paradoxe du rapport des jeunes Français à leur avenir " (2008). "Ce paradoxe renvoie en fait à la combinaison de deux optiques différentes combinant pour reprendre la formule de Spinoza "pessimisme de l'intelligence et optimisme de la volonté". "Pessimisme de l'intelligence qui passe par une conscience aigue des blocages de notre société : la réalité socio-économique; et "optimisme de la volonté " qui repose sur une confiance importante pour une grande part de la jeunesse de peser sur son destin personnel ». Anaïs (20 ans, L3 de sociologie) explore bien ce paradoxe dont parle Jean-Claude Richez : "Ma sortie des études, c'est vrai quelle me fait un peu peur déjà. Mon entrée sur le marché du travail, en fait c'est des choses que je ne visualise pas vraiment, on va dire que je l'envisage difficilement et j'espère qu'elle se passera bien. J'ai confiance en moi, je pense que jai des facultés. Je vois des abrutis qui ont des gros postes, je pense que je peux arriver à faire quelque chose plus tard. Ça me fait peur, mais je ne suis pas non plus trop angoissée. Il y a des grosses difficultés pour trouver du travail, je sais que ça ne sera pas facile, je vais maacrocher et je pense qu'en persévérant, je peux la faire cette entrée. "

\section{Une indétermination liée au fonctionnement de l'institution et au processus d'individualisation}

Comment trois années d'études n'ont-elles pas permis l'élaboration du projet professionnel et l'anticipation de l'avenir ? Plusieurs types d'explication peuvent être avancés qui sont liés à la fois au fonctionnement de l'institution universitaire et au processus contemporain de l'individualisation.

\subsection{L'université, une institution peu structurante}

L'orientation est d'autant moins aisée que le fonctionnement de l'université aujourd'hui n'aide pas les étudiants à se déterminer précisément. L'université est une institution peu structurante, "faible" pour les étudiants : elle "propose un contexte d'action où l'interdépendance entre les membres est très faible, et où l'incertitude concerne autant les objectifs et les moyens d'y parvenir, que l'appartenance même à un collectif ou un ensemble cohérent " (Felouzis, 2001, p. 15).

Afin de réduire les parcours d'échec des étudiants et leurs sorties trop nombreuses de l'enseignement supérieur sans diplôme, les universités ont pourtant développé des

2.Une enquête internationale récente a cherché à comparer les jeunesses dans le monde (Stellinger, 2008). Le jeunesse française est décrite comme plus pessimiste, désilusionnée et moins solidaire que certaines jeunesses de nos voisins européens. Ainsi, $60 \%$ des jeunes Danois estiment que leur "avenir est prometteur ", quand il sont $36 \%$ en Allemagne et seulement $26 \%$ en France. 
structures et des actions destinées à l'accompagnement des projets d'études et d'insertion des étudiants. À l'université de Tours, la MOIP (Maison de l'Orientation et de l'Insertion Professionnelle) développe de nombreuses manifestations dans ce domaine : l'organisation de la semaine de l'insertion professionnelle en novembre de chaque année ; la mise en place d'une UEL (Unité d'enseignement libre) "Préparez votre projet professionnel ", d'ateliers CV et lettres de motivations, de conférences métiers en direction des étudiants ainsi que des formations de sensibilisation à l'insertion professionnelle à destination des enseignants. Pourtant, pour les étudiants rencontrés qui n’ont pas encore déterminé leurs projets d'avenir, ce service d'orientation est, lorsqu'il est connu, sévèrement critiqué. Ses compétences, la légitimité de sa fonction sont souvent mises en doute.

Les COP (Conseillers d'Orientation Psychologues) ne sont pas épargnés. Les critiques formulées révèlent l'ambivalence des demandes étudiantes en matière d'information et d'orientation. Majoritairement, ils regrettent leur entrevue avec un conseiller d'orientation car, assurent-ils, ils n'ont rien appris et n'ont pas pu mieux choisir une orientation, un domaine d'activité après le rendez-vous. En réalité, ils ne se sentaient pas beaucoup plus avancés "Ça m’a servi à rien du tout, ça m’a plus troublé qu'autre chose » (Sophia, 20 ans, L3 d'histoire de l'Art). En fait, le malaise vient plutôt de la manière dont se joue la relation entre l'étudiant et le conseiller d'orientation, les deux protagonistes soutenant des positions qui sont, dans les récits des étudiants, toujours contradictoires. Lorsque la demande de l'étudiant était de l'aider à formaliser un projet de métier précis, la réponse du conseiller a été de valoriser la démarche autonome du projet par l'étudiant lui-même. "J'ai demandé à voir quelquiun en disant que jétais un petit peu perdue, que jétais en psycho et que je ne savais pas trop quoi faire. Et là, la personne m'a répondu 'Bah, je ne peux pas répondre à votre place!' Donc voilà, j'ai vraiment ressenti ça comme très inutile » (Coralie, 20 ans, L3 de psychologie). Dans le même ordre d'idée, l'expérience de Sandra, inscrite en L3 de sociologie, est également significative : "Ils ne torientent pas. Mis à part te comprendre, c'est tout ce quils font. 'oui, je comprends, je comprends...' Ça va bien, mais j'ai un avenir aussi à chercher. " En revanche, lorsque le conseiller propose des choix précis, bien souvent l'étudiant considère qu'ils sont "à côté de la plaque » et qu’au final, ils ont préféré choisir seuls.

Ces réactions révèlent en tout cas les difficultés rencontrées par les étudiants dans la réception de l'information lorsqu'il s'agit d'une demande d'ajustement du projet. Pour Anaïs (L3 de sociologie) les conseillers d'orientation, "c'est des gens qui en savent moins que toi sur ce que tu veux faire". Elle justifie : "J'y suis allée l'année dernière et je leur ai dit que jétais en L2 de sociologie, que jaimerais bien tenter les écoles de journalisme et que jaimais aussi la communication (...) Et ils ont vraiment répondu à côté de la plaque. (...) En gros, ils miont un petit peu découragée en fait (...). En fait, je pense quills nécoutent pas du tout la personne qu'ils ont en face d'eux, ils ne cherchent pas à la cerner."

Ces réactions révèlent plus profondément une tension entre des principes de réalité (choisir une orientation, un métier potentiellement favorable à l'insertion professionnelle) et le désir de réalisation de soi. Les conseillers d'orientation "ne sont vraiment pas là pour 
aider, c'est clair. J'ai un ami qui est dessinateur. Enfin qui dessine honnêtement, vraiment très, très bien. Il a fait des expositions déjà. Quand il est allé au CIO, ils lui ont dit clairement 'non mais monsieur, il faut redescendre sur terre. Artiste, ce n'est pas un métier'. (...) Je pense que quand on est conseiller d'orientation psychologue, il faut rendre les étudiants un peu lucides sur ce quil est possible de faire, mais pas non plus leur détruire et leur démolir complètement leurs rêves ».(Anais, L3 de sociologie)

Dans le processus de détermination de son projet professionnel, cette notion de rêve paraît fondamentale et nous invite à regarder du côté des logiques de la construction de l'identité et de la réalisation de soi pour comprendre plus profondément les hésitations de ces étudiants.

\subsection{Le processus d'individualisation ou le flou qui caractérise les cheminements}

Pourquoi certains n'arrivent-ils pas à se déterminer ? Se situant encore " au cœur " de leurs études, ces étudiants en sont au stade des interrogations. Un terme repris par quasiment l'ensemble des étudiants résume bien cette disposition : c'est le "flou ". "Alors là, en ce moment, c'est un peu, comment dire, l'incertitude, le flou total donc je ne sais pas trop encore " explique Anaïs (20 ans, L3 de sociologie) qui, par la formulation qu'elle utilise, pourrait devenir le porte-parole de ces étudiants.

Pour nous, le flou exprimé par les étudiants est le révélateur d'une tension qui se joue entre le processus d'individualisation - valorisant la logique de l'autonomie et la révélation de soi - et la logique du placement. Le processus d'individualisation est devenu aujourd'hui une dimension centrale des sociétés modernes. "Idéalement, il (l'individu) a le pouvoir - reconnu et validé socialement - de définir ses appartenances, de décider de sa vie, de résister aux évidences d'une identité que d'autres lui imposeraient" (F. de Singly, 2005, p. 68). L'auteur précise que "la jeunesse n'échappe pas à ce mouvement général de valorisation de l'indépendance et de l'autonomie " (2008, p. 6) et qu'à cette période de la vie, "le processus d'individualisation consiste à pouvoir prendre seul des décisions qui concernent sa vie » (p. 7). L'enjeu pour l'individu est de s'émanciper et de réaliser sa "vocation personnelle ". Dans ce contexte, une attention très forte est accordée à la révélation de soi et à l'épanouissement personnel que chacun souhaite atteindre par l'expérimentation individuelle. Ce processus de l'individualisation a suscité de fortes transformations dans la manière de concevoir les parcours et les âges de la vie. À ce titre, l'âge adulte ne doit plus être envisagé comme un stade à atteindre, définissant une certaine finitude des positions et du statut. S'appuyant sur un travail de recherche auprès de jeunes européens, Cécile Van de Velde note que « devenir adulte ne se conçoit plus seulement comme un statut à acquérir mais également comme un chemin à construire, dans un parcours qui se veut signifiant" (2008, p. 7). 
Sur la dimension de l'insertion professionnelle, l'enjeu pour les jeunes est ainsi de trouver une orientation et un métier ou un champ d'activités qui soient en adéquation avec soi, avec les qualités qu'on se reconnaît pour garantir cet épanouissement personnel. "Mon but, c'est d'avoir un métier qui me plaît " explique Charlotte (21 ans, L3 de psychologie). Avoir un métier qui plaît consiste à exercer dans quelque chose qui correspond aux qualités et aux envies que l'on se reconnait. "J'adore écrire, j'adore observer, j'adore regarder les gens autour. Donc c'est un petit mélange de tout ça qui me donne l'envie d'essayer de devenir journaliste " justifie Anaïs (20 ans, L3 de sociologie). La prise en compte de ses qualités et de ses envies est censée garantir l'épanouissement personnel : "Moi je veux un boulot épanouissant mais aussi calme et sans stress. Je ne veux pas me prendre la tête pour des trucs qui n'en valent pas la peine. " (Rémi, 21 ans, L3 Histoire)

Ce processus d'individualisation et cette exigence de la réalisation de soi se développent, dans le domaine des études et de l'insertion professionnelle, dans le cadre de la logique du placement, présentée précédemment. Comme évoqué, le cas français a ceci de particulier que la certification scolaire est dans notre pays un enjeu central dans la détermination du rang social qu'occupera l'individu. Les étudiants ont intériorisé le poids du diplôme, considérant "la gravité avec laquelle les jeunes français envisagent le choix des études notamment au moment de l'entrée dans le cycle supérieur : celui-ci est codé comme le moment de "choisir sa vie" "(Van de Velde, 2008, p. 138).

L'explication de ce flou se trouve dans la difficulté éprouvée par les étudiants à choisir une orientation, considérée comme définitive, et qui corresponde aux qualités qu'ils se reconnaissent. On voit ainsi dans l'indétermination du choix une certaine forme de malaise à n'avoir pas su encore se déterminer, à n'avoir pu encore se déchiffrer. Ainsi Marion (22 ans), après deux ans en LEA (langues étrangères appliquées) et trois ans en musicologie (elle est inscrite en L3 de musicologie) hésite : "Le problème, c’est que je suis intéressée par plein de choses mais justement ce n'est pas fixe en fait. " D'autant plus que dans ce processus d'individualisation, l'individu est responsable de son parcours autant pour ses réussites que pour ses échecs. Se dit également le refus de concevoir sa vie future sous un angle linéaire, attentif en cela à la réalisation de soi : "En fait, je ne me vois pas forcément faire la même chose toute ma vie, faire le même truc pendant quarante ans. Non merci! À la longue, ça doit être lassant : toujours faire la même chose pendant toute sa vie. Non je veux pouvoir faire $x$ choses, bouger, repartir de zéro, recommencer tout. " (Anaïs, 20 ans, L3 de sociologie)

En réaction au modèle de cette détermination réclamée, les étudiants esquissent ainsi un plaidoyer pour la maturation lente.

La logique du temps est ainsi privilégiée. Ces étudiants souhaitent se donner le temps pour se déterminer, pour se déchiffrer. En s'inscrivant dans un parcours scolaire dont 
la fin est encore mal dessinée, ils cherchent à se ménager " un supplément de temps ». "Le supplément de temps dont on dispose devant soi est ce qui permet vraiment de différer-et sou-

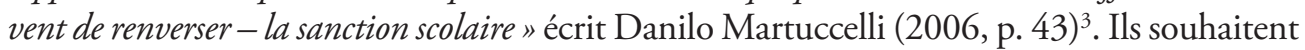
que les choses viennent petit à petit en attendant le "déclic ". Ce qui s'opère, alors, est le fait que les étudiants pourront "s'y voir ", ressentant de façon sensible que "c'est ça ". Se jouent là les éléments d'une adéquation entre ce qu'ils sont et ce qu'ils seront. Le temps deviendrait alors l'instrument du compromis entre la logique de placement et la logique de la réalisation de soi.

\section{Le doute des déterminés : entre pertinence des envies et choix pragmatique}

Dans ce contexte, comment rendre compte de l'attitude des étudiants qui ont déterminé leur choix d'études et d'insertion professionnelle ? Il serait faux de supposer qu'ils puissent être moins sensibles à la logique de la réalisation de soi et de l'épanouissement personnel. Cette dimension est également omniprésente dans leur discours et guide leurs décisions. "Je veux avoir un métier qui me plaît et dans lequel je ne miennuie pas" assure Alice (20 ans, école Polytechnique).

Ainsi cette tension entre logique du placement et logique de la révélation de soi est également agissante chez les étudiants au projet déjà établi et peut infléchir leur vision d'avenir. Si l'on a parlé de flou pour caractériser le cheminement des étudiants qui ne se sont pas encore déterminés, la notion de " doute " paraît ici la plus adaptée.

Doutes sur la pertinence de la lecture de ses envies : Sophia (20 ans, L3 Histoire de l'Art) se destine au métier de professeur des écoles, mais a "peur que ce ne soit pas le métier que j’aime vraiment, peur qu'après avoir eu le diplôme, je n'aime pas autant les enfants que ça".

Doutes sur son choix et ses implications. Le cas de Thomas (22ans), étudiant en médecine, est à cet égard très intéressant : "Ça ne veut pas dire que je suis super heureux et que tout va bien. Je regrette peut-être de ne pas avoir connu autre chose, mais maintenant que j'ai choisi, autant essayer de vivre avec ce que j’ai choisi ". À l'analyse de son entretien, on croit comprendre que les doutes de Thomas tiennent au rapport qu'il entretient avec l'idée de la vocation. Très clairement, il affirme qu'il ne ressent pas de vocation, au sens classique du terme ; qu'elle n'était pas présente quand il a choisi ses études et qu'aujourd'hui il ne sent toujours pas mû par ce sentiment. Aussi, interrogé sur les choix de son orientation vers des études de médecine, Thomas explique : "C'était par

3. Reste à savoir s'ils auront tous la possibilité familiale, économique de s'autoriser ce supplément de temps. Pour Danilo Martuccelli, cette capacité est loin d'être repartie de façon égale dans tous les milieux sociaux. 
élimination. Au début, on m'a parlé de médecine, j’ai fait 'non'. Après, on m’a parlé de tout le reste et j'ai fait 'non' aussi. Et donc, il fallait bien choisir quelque chose, donc jai pris médecine." Plus loin, il ajoute : "C'était par défaut car je n'avais envie de rien d'autres, ce n'était pas par vocation. "Aujourd'hui, cette question de la vocation le questionne toujours; en fin de rencontre, il reprend : "c'est un boulot qui demande une vocation, c'est pourquoi parfois j'ai un peu peur car je ne me sens pas l'avoir, ça va peut-être venir. " Pour l'instant, affirme-t-il, "je commence à me faire à lidée que je vais être médecin. Ce neest déjà pas mal. Ce neest déjà pas si facile à se faire à cette idée-là ». Plus qu'une logique de vocation, c'est une logique de conviction qui est ici à l'œuvre : la conviction d'avoir fait le bon choix.

\section{Conclusion}

Dans cet article, nous avons cherché à montrer comment l'existence d'une tension entre la logique de placement et la logique de la réalisation de soi pouvait jouer sur les cheminements des étudiants vers l'insertion professionnelle. En effet, d'une part, dominée par l'enjeu des études et du premier emploi, l'intégration sociale des jeunes en France s'apparente à un placement au sein d'une hiérarchie prédéfinie et où les choix initiaux sont considérés comme ayant un retentissement important sur la trajectoire à venir ; d'autre part, dans le contexte du processus d'individualisation contemporain, une très forte attention est accordée à la découverte de soi et à l'épanouissement personnel.

Cette tension définit "l'épreuve juvénile " (Dubet, 2004). "Alors que l'incertitude de la jeunesse peut être perçue comme l'extension du rìgne de la liberté et de l'authenticité personnelle", dans le même temps, "l'emprise de l'école et de la formation accentue le règne de la performance, de la mise à l'épreuve de soi, de l'accumulation de diverses formes de capitaux personnels capables d'assurer l'entrée dans la vie active en position favorable " (p. 280).

À l'aune de cette tension, on peut peut-être mieux comprendre le pessimisme des jeunes. Il apparait que les jeunes français qui déclarent avoir moins le moral sont aussi ceux qui estiment ne pas avoir réellement " la maîtrise de leur existence future. Ils ont en quelque sorte le sentiment d'une dépossession d'eux-mêmes en n'ayant pas le pouvoir sur leur vie. Cette faible maîtrise a d'importants effets négatifs, puisque ces jeunes vivent dans des sociétés dont le mot d'ordre est, par ailleurs, la responsabilité personnelle " (de Singly, 2008, p. 9). La pression scolaire telle qu'elle s'exerce en France peut ainsi donner le sentiment d'être contraint dans ses choix et dans sa liberté individuelle.

Ce constat doit nous interroger sur les types d'accompagnement vers l'insertion professionnelle à mettre en œuvre en direction des jeunes. L'attention aux logiques personnelles est pourtant présente dans le discours sur l'orientation et les actions proposées. La responsabilité de l'étudiant, défini comme un acteur qui doit "s'orienter plutôt que d'être orienté ", est attendue. Les dispositifs d'accompagnement au projet de l'étudiant, comme le Projet Personnel et Professionnel (PPP), visent cet objectif en demandant à l'individu d'être 
" entrepreneur de lui-même ». Pour autant, ces expérimentations restent inscrites dans un contexte qui valorise toujours les déterminations précoces et les parcours tubulaires entre choix d'études et choix de métier.

\section{bibliographie}

Bidart C. (2006), "Crises, décisions et temporalités : autour des bifurcations biographiques " Cahiers internationaux de sociologie, vol. 1, n 120, pp. 29-57.

Dubet F. (2004), "La jeunesse est une épreuve », in Comprendre les jeunes, Revue de philosophie et de sciences sociales, $\mathrm{n}^{\circ} 5$, pp. 275-291.

Erlich V. et Verley E. (2010), «Une relecture sociologique des parcours des étudiants français : entre segmentation et professionnalisation ", Education et sociétés, n 26, 2010/2, pp. 71-88.

Felouzis G. (2001), La condition étudiante. Sociologie des étudiants et de l'université, PUF, Paris, 300 p.

Galland O. (2009), Les jeunes français ont-ils raison d'avoir peur?, Armand Colin, Paris, $160 \mathrm{p}$.

Giret J.-F. (2009), « L'évolution des conditions d'insertion professionnelle des étudiants ", in Gruel L.Galland O. et Houzel G. (dir), Les étudiants en France. Histoire et sociologie d'une nouvelle jeunesse, PUR, Rennes, pp. 331-348.

Martuccelli D. (2006), Forgé par l'épreuve. L'individu dans la France contemporaine, Paris, Armand Colin, 478 p.

Richez J.-C. (2008), Les jeunes face à leur avenir saisis par les sondeurs : pessimisme de l'intelligence ou optimisme de la volonté ? Note de synthèse relative à l'étude de la Fondation pour l'Innovation Politique « Les jeunes face à leur avenir ".

Singly de F. (2008), "Préface : les jeunesses du monde moderne ", in Les jeunesses face à leur avenir. Une enquête internationale, sous la direction d'Anna Stellinger, Fondation pour l'Innovation Politique, Paris, pp. 5-15.

Singly de F. (2005), "Les disparitions de l'individu singulier en sociologie ", in Politiques de l'individualisme, entre sociologie et philosophie, sous la direction de Philippe Corcuff, Jacques Ion et François de Singly, Les éditions Textuel, Paris, pp. 63-86.

Stellinger A. (dir) (2008), Les jeunesses face à leur avenir. Une enquête internationale, Fondation pour l'Innovation Politique, Paris, $187 \mathrm{p}$.

Van de Velde C. (2008), Devenir adulte. Sociologie comparée de la jeunesse en Europe, coll. «Le lien social », PUF, Paris, 278 p. 